\title{
Minimally Invasive Esophagectomy: Are There Significant Benefits?
}

\author{
Benedetto Mungo $\cdot$ Daniela Molena
}

Published online: 27 May 2014

(c) Springer Science + Business Media New York 2014

\begin{abstract}
The incidence of esophageal cancer and, more specifically, of esophageal adenocarcinoma, continues to increase in the United Sates (US), mostly affecting white male patients. The mainstay of curative treatment for localized disease is surgery, typically after neoadjuvant chemoradiotherapy. However, esophagectomy remains a procedure burdened by significant rates of mortality and morbidity. Over the last two decades minimally invasive techniques have been adapted to esophageal resection, with the goal of improving postoperative outcomes without compromising oncological success. Numerous reports have documented the safety and feasibility of minimally invasive esophagectomy (MIE), and several centers have gained considerable experience in the technique and gathered a large case series. The overall advantage of MIE over open techniques in regards to improved outcomes and cost effectiveness remains controversial. The aim of the present work is to review the key aspects of MIE and compare the results with open surgery.
\end{abstract}

Keywords Minimally invasive esophagectomy . Outcomes of esophagectomy - Transhiatal esophagectomy . Ivor Lewis esophagectomy · 3-Field esophagectomy · Esophageal cancer · Laparoscopy · Thoracoscopy

This article is part of the Topical Collection on Esophageal/Reflux Surgery.

B. Mungo $\cdot$ D. Molena $(\square)$

Division of Thoracic Surgery, Department of Surgery, Johns

Hopkins University School of Medicine, 600 N Wolfe Street,

Blalock 240, Baltimore, MD 21287, USA

e-mail: dmolena2@jhmi.edu

B. Mungo

e-mail: bmungo1@jhmi.edu

\section{Introduction}

Esophageal cancer affects more than 450,000 individuals worldwide and the incidence continues to increase [1]. Estimates from the National Cancer Institute reported that in the US this condition affected 17,990 individuals during 2013 and was the cause of 15,210 deaths [2]. The most common esophageal cancer subtype in the US is adenocarcinoma, affecting predominantly white overweight males, while squamous esophageal cancer remains the most prominent histologic type worldwide [3•]. Even though ongoing advancements in medical and surgical treatment have improved the prognosis for this condition, the overall 5-year survival rate of patients with resectable esophageal cancer remains disappointing, ranging from 15 to $34 \%[4 \cdot 5]$. Surgery is the mainstay of treatment for esophageal cancer, whether it be for patients in an early stage of the disease, or after neoadjuvant concurrent chemotherapy and radiation in patients with more advanced locoregional involvement [6••]. Esophagectomy remains one of the most complex surgical procedures and it is associated with high morbidity and mortality rates; pulmonary complications account for the most frequent postoperative adverse event (20-40\% of all patients) [7]. The mortality rate for esophagectomy in the US has been reported as high as $23 \%$, but in most high-volume medical centers it ranges from 1 to $5 \%$ [8].

After the first thoracoscopic esophagectomy, described by Cuschieri et al. [9] in 1992, several minimally invasive techniques have been developed in an effort to improve post-operative outcomes. Many centers around the world have adopted minimally invasive esophagectomy (MIE) and some authors have reported a large case series [10•]. However, the advantages of MIE over open esophagectomy are still debated. 
The aim of the present work is to review the key aspects of MIE and compare outcomes with open esophagectomy.

\section{Surgical Techniques}

Transhiatal, Ivor Lewis and McKeown esophagectomy, the three major approaches for open esophagectomy, have all been performed using minimally invasive techniques. In addition, a broad variety of modified, hand-assisted and hybrid approaches have been described as well.

The laparoscopic transhiatal approach was first developed by DePaula et al. [11] in 1995; the authors described the technique for this procedure and reported lower postoperative complication rates than those described in the literature for open transhiatal esophagectomy.

Similar to the open approach, transhiatal MIE potentially leads to fewer respiratory complications by avoiding direct thoracic access and lung atelectasis. Furthermore, by avoiding patient's repositioning during the procedure, this approach benefits from a shorter operative time [12•]. However, this comes at the cost of a poorer visualization of the intrathoracic esophagus and minimal lymphatic dissection in the thorax [13]. Data from one of the largest studies comparing laparoscopic with open transhiatal esophagectomies showed that transhiatal MIE was associated with a shorter hospital and intensive care unit stay, with similar operative time and morbidity [14]. Also of note, the magnified visualization offered by the laparoscopic camera may allow for less 'blind' mediastinal dissection, therefore reducing blood loss. This is particularly relevant in transhiatal MIE compared to other MIE techniques that involve direct thoracic visualization [13].

The Ivor Lewis esophagectomy is the most common approach for esophagectomy in the western world [15•]. This is not surprising considering that adenocarcinomas of the lower esophagus are the most prevalent subtype in this geographical area. Ivor Lewis esophagectomy is a particularly well suited technique for these tumors, which often don't require a high esophageal transection but rather lead to extended gastric resections that may result in a short conduit, far from ideal for a neck anastomosis [15•].

Among the advantages of an Ivor Lewis esophagectomy are good exposure of the thorax via direct esophageal visualization, and access for extensive mediastinal lymphadenectomy. However, with an open approach there is an element of "conflict of interest" in that a high (5th interspace) thoracotomy is beneficial for placing the anastomosis above the azygous arch to minimize reflux complications, but a lower ( 7 th interspace) thoracotomy allows better exposure for a lymph node dissection around the area where most adenocarcinomas are located. Another advantage of the Ivor Lewis approach is that a more extensive gastric resection is possible since the conduit does not need to reach to the neck. The anastomosis can therefore be performed using a segment of stomach with a richer vascular supply, leading to a lower anastomotic leak rate [16, 17]. Finally, this approach is associated with fewer incidences of recurrent laryngeal nerve injury and therefore less postoperative aspiration risk. The downsides of a transthoracic approach include the need for single lung ventilation, atelectasis and respiratory compromise, and potentially harmful sequelae of an intrathoracic anastomotic leak [18, 19].

The first description of a complete minimally invasive approach to an Ivor Lewis esophagectomy was reported in 1999 when Watson et al. [20] described a totally laparoscopic and thoracoscopic technique for an esophagectomy with an intrathoracic hand-sewn anastomosis. With the evolution of the MIE techniques, a circular mechanical anastomosis, with the anvil introduced in the proximal esophagus either through a transthoracic or transoral route, has been largely preferred to the hand-sewn anastomosis [21-23]. Stapled anastomoses have been reported to yield a lower rate of anastomotic leakage and stenosis [17].

A recent study comparing open and MIE using the Ivor Lewis approach showed no difference in operative time and cardiac complications [7]. However, MIE was associated with significantly lower rates of respiratory complications, intraoperative blood loss and intravenous fluid infusion and shorter stays in the intensive care unit (ICU) and hospital overall. Moreover there was no difference when comparing the oncological outcomes (number of lymph nodes removed and resection margins). Not all authors, however, have reported similar results: Noble et al. [15] showed no difference between the open and MIE Ivor Lewis procedure in terms of length of stay (LOS), overall morbidity and pulmonary complications, while blood loss was shown to remain lower for MIE.

The three-field esophagectomy procedure was originally described by McKeown [24]: the open technique allows for a complete esophagectomy with extensive lymph nodal dissection and cervical anastomosis through a combined approach involving a thoracotomy, a laparotomy and a cervical incision. Advantages of this approach include the ability to optimally position the thoracotomy for the area of nodal dissection based on the tumor location and a better proximal margin in patients with upper third squamous esophageal cancers. Other reported advantages include a reduced incidence of reflux symptoms and less morbidity with an anastomotic leak compared to an intra-thoracic anastomosis [25, 26]. However, this procedure involves three areas of the body and has potential disadvantages related to both thoracic and a cervical access. In general, the McKeown esophagectomy is a good choice when a total esophageal resection is needed, and also for high and mid-thoracic esophageal cancers, patients with an extended 
long-segment Barrett's esophagus and for benign disorders (end-stage achalasia, complex benign strictures) [27]. Luketich et al. [28] were among the first authors to develop and describe a minimally invasive technique for the McKeown esophagectomy in 1998. Nguyen et al. [29] compared three-hole MIE to open esophagectomy and reported that MIE was associated with lower operative time, less blood loss and shorter ICU and hospital stays. Nevertheless, the incidence rates of postoperative anastomotic leaks and respiratory complications were comparable between the two approaches. The initial Pittsburgh experience, evaluating a series of 222 patients who underwent MIE (mostly with the minimally invasive three-hole technique), showed the procedure to be not only safe but also associated with a lower mortality rate and shorter hospital stay compared to the previously-published open series [30]. Recent work by Chen et al. [31] reported that a McKeown MIE is a safe and feasible procedure leading to surgical and oncological outcomes comparable with those of its open counterpart.

\section{Surgical Outcomes}

A large number of systematic reviews, meta-analyses and case-series have reported contradictory results for surgical outcomes of minimally invasive esophagectomy (e.g., mortality, respiratory complications, length of stay, anastomotic leak rate). Cash et al. [13] recently emphasized this controversy stating that although systematic reviews of MIE have shown equivocal results, meta-analyses have suggested that MIE leads to lower morbidity with comparable mortality. Many factors might contribute to this plurality of findings: for example, MIE is a technically challenging procedure, requiring advanced minimally invasive surgical skills and a considerably long learning curve (for thoracoscopic esophagectomy, the plateau is reached after no less than 30 cases) [32, 33•]. Therefore, large single center studies reporting on procedures performed by experienced surgeons are likely to display better results with lower mortality and morbidity rates and shorter lengths of stay.

In a recent review by Kim et al. [34] summarizing data from an institutional series, case-control studies and systematic reviews, the authors concluded that while operative mortality is not significantly different between the two approaches there may be less morbidity with the MIE approach.

In their critical review, Sudarshan and Ferri [23] provided more detailed insight on several specific surgical outcomes; they reported no striking differences between MIE and open surgery in terms of in-hospital mortality, pulmonary complications and leak rates. Open surgery was associated with shorter operative times and fewer reoperations. On the other hand, MIE showed a trend towards less blood loss and shorter length of ICU and hospital stays.
A finding of concern in a number of early MIE reports was a higher than expected rate of gastric tube necrosis [35, 36]. It was postulated by some that this was perhaps related to the laparoscopic technique or pneumoperitoneum. This hypothesis appeared to be supported by findings in other studies, such as the series by Crenshaw et al. [37] in which a significant decrease in gastric conduit failure was observed when the conduit was formed extracorporeally. However, factors other than the minimally invasive approach itself are more likely to contribute to gastric tube necrosis: a narrow tube, for example, was reported to be more prone to gastric tip necrosis [30]. In addition, a recent study hypothesized that gastric tube necrosis is a learning curve issue, pointing out how an increase in operative experience and meticulous attention during key steps of the procedure correlates with a reduction in the occurrence of this event [38].

Thus far only one randomized controlled trial comparing MIE to open esophagectomy has been published [39••]. The study, which was carried out at five centers in three countries, enrolled 56 patients in the open group and 59 in the MIE group. Pulmonary infection within the first 2 weeks after surgery and during the whole hospital stay was the primary outcome of this trial. The authors reported that MIE resulted in improved short-term outcome in terms of decreased pulmonary infections, a shorter hospital stay, and a better quality of life, while yielding a similar oncologic outcome to open surgery. A minimally invasive approach may be associated with less immunosuppression than open esophagectomy resulting in better preserved leukocyte counts, IL-8 levels and stress response, all factors that may contribute to fewer post-operative respiratory infections in the MIE group [40]. However, of note is the technique used for MIE in this trial involved prone positioning during the thoracoscopic phase; this might have positively affected the pulmonary outcomes given the absence of single-lung ventilation and lung atelectasis.

Some potential limitations of this trial should be pointed out such as a modest sample size and a relative lack of experience with MIE for surgeons in the minimally invasive group [41].

\section{Oncological Outcomes}

Oncological equivalence between MIE and open surgery for esophageal cancer is another topic of debate. The variety of approaches used for both MIE and open procedures, as well as the difference in neo-adjuvant and adjuvant protocols, add complexity to the analysis of oncological outcomes. Dantoc et al. [42] performed a systematic review of the literature up to 2011 and found no difference in the 5-year survival rates of MIE and open esophagectomy. In the same year results from a meta- 
analysis retrieving data from separate case-control studies confirmed no difference in oncological outcomes between open and MIE approaches [43•]. More recently Dolan et al. [44] reported on oncological equivalence of open esophagectomy and MIE describing comparable long-term survival rates and, in addition, a higher lymph node harvest rate in the MIE group. The latter finding might be explained by the advantage provided via the improved magnified visualization of the laparoscopic/thoracoscopic camera during lymphadenectomy.

The literature is contradictory regarding the adequacy of the resected margins; in fact, some authors describe no difference between minimally invasive and open patients, while others demonstrate a slightly higher rate of positive margins with MIE [7, 23, 45].

In order to analyze oncological outcomes with a higher level of evidence additional randomized controlled studies would certainly be required.

\section{Post-Surgical Recovery}

The major advantages of minimally invasive surgery have been extensively demonstrated for surgical procedures such as cholecystectomy and Nissen fundoplication and include a shorter hospital stay, faster recovery and a better early post-operative quality of life. Parameswaran et al. [46] compared recovery after MIE (hybrid and open esophagectomy) by assessing fatigue levels and how soon patients returned to daily living activities as indicators of recovery. While immediately after the procedure increased fatigue levels and a decreased ability to perform daily activities were observed for every technique, these parameters started to improve considerably earlier and faster after MIE compared to open an esophagectomy. This correlates well with other studies that document significant improvement in role functioning and pain in the MIE group after 1-3 months from surgery. Technique-associated disparities in health-related quality of life, however, seem to disappear by one year postoperatively [47].

\section{Cost Analysis}

Cost effectiveness of MIE is, again, controversial: while supporters of MIE advocate that fast recovery and shorter LOS compensate for higher operative costs, other authors suggest that enhanced recovery pathways can further lower the costs of open esophagectomy, making it an overall cheaper option. Literature on the topic includes discordant reports, and generalizations on cost analysis are difficult given the differences in costs across health systems of disparate countries. A 2013 study by Lee et al. [48] concluded that MIE proved to be advantageous over open resection by resulting in decreased costs and increased quality-adjusted life years. Moreover, the authors reasonably postulated that extending the analysis to productivity losses and caregiver burden, in a societal perspective, would likely further increase the cost-effectiveness of MIE.

In work by Parameswaran et al. [49] MIE resulted in a higher operative cost due to the need for specialized and disposable equipment, but slightly lower inpatient care costs: this resulted in overall comparable costs for MIE and open esophagectomy. Other authors, however, have shown that a shorter LOS after MIE is not enough to offset significantly higher operating theatre costs [50].

\section{Conclusions}

Since its introduction, MIE has gained increasing popularity and at the same time has been the core of a fervent debate. While holding promise, it has not replaced the open procedure as has been the case for most patients undergoing less complex procedures such as a cholecystectomy or Nissen fundoplication. The continued evolution of this technology will undoubtedly stimulate improvement and refinement of MIE allowing for progressively better outcomes. In parallel, the increasing familiarity with minimally invasive techniques among the new generation of surgeons will further promote and increase the use of MIE. Currently, however, the variable disease characteristics as well as the many technical variants and modifications of MIE make an objective evaluation of the results very difficult. Nonetheless, we strongly believe that in the right patient MIE has the potential to yield precious advantages, like any other minimally invasive procedure, in terms of decreased pain, less morbidity and faster recovery, while not compromising the oncologic outcome in patients with cancer.

\section{Compliance with Ethics Guidelines}

Conflict of Interest Benedetto Mungo and Daniela Molena declare that they have no conflict of interest.

Human and Animal Rights and Informed Consent This article does not contain any studies with human or animal subjects performed by any of the authors.

\section{References}

Papers of particular interest, published recently, have been highlighted as:

- Of importance

•- Of major importance

1. Pennathur A, Gibson MK, Jobe BA, Luketich JD. Oesophageal carcinoma. Lancet. 2013;381(9864):400-12. 
2. National Cancer Institute: estimated new cases and deaths from esophageal cancer in the United States in 2013. Available at: http://www.cancer.gov/cancertopics/types/esophageal. Accessed 17 Jan 2013.

3. - Zhang J, Wang R, Liu S, Luketich JD, Chen S, Chen H, et al. Refinement of minimally invasive esophagectomy techniques after 15 years of experience. J Gastrointest Surg. 2012;16(9): 1768-74. Description of evolution of MIE technique at one of the most experienced centers with this approach.

4. - Sjoquist KM, Burmeister BH, Smithers BM, Zalcberg JR, Simes RJ, Barbour A, et al. Survival after neoadjuvant chemotherapy or chemoradiotherapy for resectable oesophageal carcinoma: an updated meta-analysis. Lancet Oncol. 2011;12(7):681-92. Updated large meta-analysis studying the effects of neoadjuvant treatment in patients with esophageal cancer.

5. Taylor MD, LaPar DJ, Davis JP, Isbell JM, Kozower BD, Lau $\mathrm{CL}$, et al. Induction chemoradiotherapy and surgery for esophageal cancer: survival benefit with downstaging. Ann Thorac Surg. 2013;96(1):225-31.

6. • van Hagen P, Hulshof MC, van Lanschot JJ, Steyerberg EW, van Berge Henegouwen MI, Wijnhoven BP, et al. Preoperative chemoradiotherapy for esophageal or junctional cancer. N Engl J Med. 2012;366(22):2074-84. Randomized controlled trial (CROSS trial) reporting on the benefits of induction therapy for esophageal cancer.

7. Sihag S, Wright CD, Wain JC, Gaissert HA, Lanuti M, Allan JS, et al. Comparison of perioperative outcomes following open versus minimally invasive ivor lewis oesophagectomy at a single, high-volume centre. Eur J Cardiothorac Surg. 2012;42(3):430-7.

8. Birkmeyer JD, Siewers AE, Finlayson EV, Stukel TA, Lucas FL, Batista I, et al. Hospital volume and surgical mortality in the US. N Engl J Med. 2002;346(15):1128-37.

9. Cuschieri A, Shimi S, Banting S. Endoscopic oesophagectomy through a right thoracoscopic approach. J R Coll Surg Edinb. 1992;37(1):7-11.

10. - Luketich JD, Pennathur A, Awais O, Levy RM, Keeley S, Shende M, et al. Outcomes after minimally invasive esophagectomy: review of over 1,000 patients. Ann Surg. 2012;256(1): 95-103. Outcomes from the largest single center series with minimally invasive esophagectomy.

11. DePaula AL, Hashiba K, Ferreira EA, de Paula RA, Grecco E. Laparoscopic transhiatal esophagectomy with esophagogastroplasty. Surg Laparosc Endosc. 1995;5(1):1-5.

12. Bhayani NH, Gupta A, Dunst CM, Kurian AA, Reavis KM, Swanstrom LL. Esophagectomies with thoracic incisions carry increased pulmonary morbidity. JAMA Surg. 2013;148(8):733-8. Analysis of a surgical, national research oriented database comparing the pulmonary and overall morbidity of esophagectomies with and without thoracic incisions.

13. Cash JC, Zehetner J, Hedayati B, Bildzukewicz NA, Katkhouda $\mathrm{N}$, Mason RJ, et al. Outcomes following laparoscopic transhiatal esophagectomy for esophageal cancer. Surg Endosc. 2013;27(4):1260-6.

14. Maas KW, Biere SS, Scheepers JJ, Gisbertz SS, van-der-Peet DL, Cuesta MA. Laparoscopic versus open transhiatal esophagectomy for distal and junction cancer. Rev Esp Enferm Dig. 2012;104(4):197-202.

15. - Noble F, Kelly JJ, Bailey IS, Byrne JP, Underwood TJ, South Coast Cancer Collaboration-Oesophago-Gastric (SC3-OG). A prospective comparison of totally minimally invasive versus open ivor lewis esophagectomy. Dis Esophagus. 2013;26(3):263-71. Prospective single-center cohort study comparing patients undergoing elective 2 stage MIE or open Ivor Lewis esophagectomy, delineating some of the advantages of MIE.

16. Nguyen NT, Follette DM, Lemoine PH, Roberts PF, Goodnight JE Jr. Minimally invasive ivor lewis esophagectomy. Ann Thorac Surg. 2001;72(2):593-6.
17. Maas KW, Biere SS, Scheepers JJ, Gisbertz SS, Turrado Rodriguez VT, van der Peet DL, et al. Minimally invasive intrathoracic anastomosis after ivor lewis esophagectomy for cancer: a review of transoral or transthoracic use of staplers. Surg Endosc. 2012;26(7):1795-802.

18. Hsu PK, Huang CS, Wu YC, Chou TY, Hsu WH. Open versus thoracoscopic esophagectomy in patients with esophageal squamous cell carcinoma. World J Surg. 2013;37(12):2740-6.

19. Bizekis C, Kent MS, Luketich JD, Buenaventura PO, Landreneau $\mathrm{RJ}$, Schuchert MJ, et al. Initial experience with minimally invasive ivor lewis esophagectomy. Ann Thorac Surg. 2006;82(2): $402-6$.

20. Watson DI, Davies N, Jamieson GG. Totally endoscopic ivor lewis esophagectomy. Surg Endosc. 1999;13(3):293-7.

21. Campos GM, Jablons D, Brown LM, Ramirez RM, Rabl C, Theodore P. A safe and reproducible anastomotic technique for minimally invasive ivor lewis oesophagectomy: the circular-stapled anastomosis with the trans-oral anvil. Eur J Cardiothorac Surg. 2010;37(6):1421-6.

22. Zhang RQ, Xia WL, Kang NN, Ge W, Chen AG, Zhu KC. Purse string stapled anastomotic technique for minimally invasive ivor lewis esophagectomy. Ann Thorac Surg. 2012;94(6):2133-5.

23. Sudarshan M, Ferri L. A critical review of minimally invasive esophagectomy. Surg Laparosc Endosc Percutan Tech. 2012;22(4): $310-8$.

24. McKeown KC. Total three-stage oesophagectomy for cancer of the oesophagus. Br J Surg. 1976;63(4):259-62.

25. Swanson SJ, Sugarbaker DJ. The three-hole esophagectomy. The brigham and women's hospital approach (modified McKeown technique). Chest Surg Clin N Am. 2000;10(3):531-52.

26. Swanson SJ, Batirel HF, Bueno R, Jaklitsch MT, Lukanich JM, Allred E, et al. Transthoracic esophagectomy with radical mediastinal and abdominal lymph node dissection and cervical esophagogastrostomy for esophageal carcinoma. Ann Thorac Surg. 2001;72(6):1918-24.

27. Perry Y, Fernando HC. Three-field minimally invasive esophagectomy: current results and technique. J Thorac Cardiovasc Surg. 2012;144(3):S63-6.

28. Luketich JD, Nguyen NT, Weigel T, Ferson P, Keenan R, Schauer P. Minimally invasive approach to esophagectomy. JSLS. 1998;2(3):243-7.

29. Nguyen NT, Follette DM, Wolfe BM, Schneider PD, Roberts P, Goodnight JE Jr. Comparison of minimally invasive esophagectomy with transthoracic and transhiatal esophagectomy. Arch Surg. 2000;135(8):920-5.

30. Luketich JD, Alvelo-Rivera M, Buenaventura PO, Christie NA, McCaughan JS, Litle VR, et al. Minimally invasive esophagectomy: outcomes in 222 patients. Ann Surg. 2003;238(4):486-94.

31. Chen B, Zhang B, Zhu C, Ye Z, Wang C, Ma D, et al. Modified McKeown minimally invasive esophagectomy for esophageal cancer: a 5-year retrospective study of 142 patients in a single institution. PLoS ONE. 2013;8(12):e82428.

32. Osugi H, Takemura M, Higashino M, Takada N, Lee S, Ueno M, et al. Learning curve of video-assisted thoracoscopic esophagectomy and extensive lymphadenectomy for squamous cell cancer of the thoracic esophagus and results. Surg Endosc. 2003;17(3):515-9.

33. • Guo W, Zou YB, Ma Z, Niu HJ, Jiang YG, Zhao YP, et al. One surgeon's learning curve for video-assisted thoracoscopic esophagectomy for esophageal cancer with the patient in lateral position: how many cases are needed to reach competence? Surg Endosc. 2013;27(4):1346-52. Interesting study on the learning curve needed to master this highly challenging procedure.

34. Kim T, Hochwald SN, Sarosi GA, Caban AM, Rossidis G, BenDavid K. Review of minimally invasive esophagectomy and current controversies. Gastroenterol Res Pract. 2012;25(6): 741-52. 
35. Berrisford RG, Wajed SA, Sanders D, Rucklidge MW. Shortterm outcomes following total minimally invasive oesophagectomy. Br J Surg. 2008;95(5):602-10.

36. Allum WH, Blazeby JM, Griffin SM, Cunningham D, Jankowski JA, Wong R, et al. Guidelines for the management of oesophageal and gastric cancer. Gut. 2011;60(11):1449-72.

37. Crenshaw GD, Shankar SS, Brown RE, Abbas AE, Bolton JS. Extracorporeal gastric stapling reduces the incidence of gastric conduit failure after minimally invasive esophagectomy. Am Surg. 2010;76(8):823-8.

38. Ramage L, Deguara J, Davies A, Hamouda A, Tsigritis K, Forshaw M, et al. Gastric tube necrosis following minimally invasive oesophagectomy is a learning curve issue. Ann R Coll Surg Engl. 2013;95(5):329-34.

39. •• Biere SS, van Berge Henegouwen MI, Maas KW, Bonavina L, Rosman C, Garcia JR, et al. Minimally invasive versus open oesophagectomy for patients with oesophageal cancer: a multicentre, open-label, randomised controlled trial. Lancet. 2012;379(9829):1887-92. The only randomized trial comparing MIE with open esophagectomy; high level evidence of advantages granted by MIE.

40. Maas KW, Biere SS, van Hoogstraten IM, van der Peet DL, Cuesta MA. Immunological changes after minimally invasive or conventional esophageal resection for cancer: a randomized trial. World J Surg. 2013;5(4):73-134.

41. Law S. Is minimally invasive preferable to open oesophagectomy? The Lancet. 2012;379(9829):1856-8.

42. Dantoc MM, Cox MR, Eslick GD. Does minimally invasive esophagectomy (MIE) provide for comparable oncologic outcomes to open techniques? A systematic review. J Gastrointest Surg. 2012;16(3):486-94.
43. - Dantoc M, Cox MR, Eslick GD. Evidence to support the use of minimally invasive esophagectomy for esophageal cancer: a meta-analysis. Arch Surg. 2012;147(8):768-76. Updated large meta-analysis demonstrating the advantages granted by MIE.

44. Dolan JP, Kaur T, Diggs BS, Luna RA, Schipper PH, Tieu BH, et al. Impact of comorbidity on outcomes and overall survival after open and minimally invasive esophagectomy for locally advanced esophageal cancer. Surg Endosc. 2013;27(11):4094-103.

45. Sundaram A, Geronimo JC, Willer BL, Hoshino M, Torgersen Z, Juhasz A, et al. Survival and quality of life after minimally invasive esophagectomy: a single-surgeon experience. Surg Endosc. 2012;26(1):168-76.

46. Parameswaran R, Titcomb DR, Blencowe NS, Berrisford RG, Wajed SA, Streets CG, et al. Assessment and comparison of recovery after open and minimally invasive esophagectomy for cancer: an exploratory study in two centers. Ann Surg Oncol. 2013;20(6):1970-7.

47. Darling GE. Quality of life in patients with esophageal cancer. Thorac Surg Clin. 2013;23(4):569-75.

48. Lee L, Sudarshan M, Li C, Latimer E, Fried GM, Mulder DS, et al. Cost-effectiveness of minimally invasive versus open esophagectomy for esophageal cancer. Ann Surg Oncol. 2013;20(12):3732-9.

49. Parameswaran R, Veeramootoo D, Krishnadas R, Cooper M, Berrisford R, Wajed S. Comparative experience of open and minimally invasive esophagogastric resection. World J Surg. 2009;33(9):1868-75.

50. Dhamija A, Dhamija A, Hancock J, McCloskey B, Kim AW, Detterbeck FC, et al. Minimally invasive oesophagectomy more expensive than open despite shorter length of stay. Eur J Cardiothorac Surg. 2013;44(4):637-42. 